\title{
Effect of NPK on Plant Growth and Quality of Lilium Hybrid (Asiatic Lily) Tresor under Polyhouse and Open Condition
}

\author{
Pooja Pahare $^{1 *}$ and Suchismita Mishra ${ }^{2}$ \\ Department of floriculture and landscaping, Odisha University of Agriculture and \\ Technology, Bhubaneswar 751003, India \\ *Corresponding author
}

\begin{tabular}{|l|}
\hline K e y w o r d s \\
Lilium (hybrid \\
tresor) growth, \\
Quality, Vase life, \\
NPK
\end{tabular}

\section{A B S T R A C T}

\section{Introduction}

Lilium is one of the most important genera of cut flower and pot plant production. It has always played a special role in the garden design due to the great number of species that can be adapted to many environments of garden. Moreover, the flower colors, the scents and the cultivar choice can guarantee a continuous flowering from spring to autumn. The use of Lilium cultivars for urban decoration can also be of great interest 
(Grassotti et al., 2011). Lilies occupy the fourth position after rose, tulip and spray chrysanthemum for total sales and are considered one of the leading geophytes. There exist two groups, viz. Asiatic and Oriental hybrids. Lily is the common English name for flowering plants of the Lilium genus and they are extensively being grown in greenhouses as cut favoured in global flower trade due to wider choice of growing periods, array of colours and everlasting quality. (Sindhu and Pathania, 2003) Lilium (members of which are true lilies) is a genus of herbaceous flowering plants growing from bulbs, all with large prominent flowers. Lilies are a group of flowering plants which are important in culture and literature in much of the world. Most species are native to the temperate northern hemisphere, though their range extends into the northern subtropics. Many other plants have "lily" in their common name but are not related to true lilies. The flowers are large, often fragrant, and come in a range of colours including whites, yellows, oranges, pinks, reds and purples. Markings include spots and brush strokes. The plants are late spring- or summer-flowering. Cut flowers have complex nature that requires special attention in developing handling technique, concentration of sugar and other substances for pulsing and bud opening of cut flower.

Use of floral preservatives at all stages of flower handling and marketing known to improve the flower quality, longevity and better consumer acceptability. Prolonging the vase-life depends on water balance and retardation of petal senescence which can be achieved by the use of sucrose, nutrients and certain chemicals (Beura and Singh, 2002. Nitrogen (N) has the quickest most pronounced effect on the plant growth that ultimately leads to good yield nitrogen is the constituent of different amino acids, proteins and chlorophyll which is essential for good growth of plant. Nitrogen deficiency can be characterized by stunted growth; flowering and fruiting are also reduced. (Bankar et al., 1990) Phosphorus (P) is one of the essential elements for plant growth and development the phosphorus plays on important role in maintaining quality of the flower. It is essentially required for the proper root growth the phosphorus should the applied as the basal dose. Potassium $(\mathrm{K})$ is needed for healthy roots and stems, and aids plants with the respiration process. It is sometimes called potash (Parekh et al., 2010).

The main objectives of this study include to find out the most suitable treatment for plant growth, flower yield and quality of Lilium. And to find out the suitability of Lilium under polyhouse \& open condition. Also to work out the economics of different treatments and to identify the most economic Treatment.

\section{Materials and Methods}

The present experiment was conducted in the Department of Floriculture and Landscaping, RKVY project at Biotechnology-cum-Tissue Culture Centre, OF College of Agriculture, Orissa University of Agriculture and Technology, Bhubaneswar during the year 2016. Crop lilum asiatic hybrid Tresor. Disease free healthy and fungicide (bavistin $2 \mathrm{~g} / \mathrm{l}$ of water for 30 mintes) treated bulbs are sown in the polyhouse field. After sowing the bulbs were pressed gently and given light irrigation. Planting was done on the $2^{\text {nd }}$ October, 2016. Plot with dimension $48 \mathrm{~m}^{2}$ were prepared at a spacing of $40 \mathrm{X} 40 \mathrm{~cm}$. plant nutrient (Nitrogen, Phosphorus and Potash ) each at three concentrations viz, (10:10:10) and control ((FYM mixed to the soil @ 1 $\mathrm{m}^{3}$ per $100 \mathrm{~m}^{2}$ area) were allocated in randomized block design with three replication with six bulbs per replication. The nutrient was applied in form of foliar spray and soil drenching, once at 30 days after 
planting (DAS) bulbs \& again after 30days of first application. Cultural operations like hoeing was manually taken at 30 days interval till the crop in the field. Weeding was done at monthly interval. The experimental plot was kept weed free by manual weeding till the crop in the field were followed as per standard cultural practices. Data on different growth, flowering and yield parameters were recorded on two randomly taken competitive plants for all the observations, i.e. plant height, number of functional leaves per plant, Plant spread, Days to bud emergence, Number of bud per plant, Flower Spike length, Flower Spike diameter, Flower diameter, Bud length, Bud diameter, Flower length, First flower senescence and Vase life of flower were recorded at 30 days intervals from planting to flower buds.

\section{Results and Discussion}

The results obtained from the present investigation as well relevant discussion have been summarized under following heads:

\section{Growth characters}

The data on plant height is presented in (Table $1 \mathrm{a}, \mathrm{b}$ ). A perusal of data in Table 1a shows that maximum plant height was recorded In Polyhouse maximum plant height $(11.21 \mathrm{~cm})$ at $30 \mathrm{DAP}$, was recorded with treatment Arya et al., (2010) reported that the $150 \mathrm{~kg} / \mathrm{ha}$ nitrogen, $100 \mathrm{~kg} / \mathrm{ha}$ phosphorus and $80 \mathrm{~kg} / \mathrm{ha}$ potassium were found suitable doses for the commercial cultivation of African marigold western plain. Birade et al., (2003) reported that the effects of graded levels of NPK (00:00:00; 100:75:50; 150:100:75; and 200:125:100 kg/ha.) on the growth, quality and flower production of China Aster. Were found to be beneficial for profuse growth, quality and flower production of China aster. Devi et al., (2010) reported that the effect of different nitrogen levels resulted in superior growth, flowering and yield of bulbs. Nitrogen at $220 \mathrm{~kg} / \mathrm{ha}$ gave maximum number of leaves' plant, number of tillers/plant, plant height, number of spikes/plant, spike length, rachis length, number of florets/spike, duration of flowering, number of bulbs/clump and weight of bulbs/clump and was at par with that of treatment $200 \mathrm{~kg} \mathrm{~N} / \mathrm{ha}$.

Gani et al., (2007) reported that the three levels of each $\mathrm{N}(0,50$ and $80 \mathrm{Kg} / \mathrm{ha})$ and P2O5 (0. 40 and $60 \mathrm{Kg} / \mathrm{ha}$ ) to find out the optimum doses of $\mathrm{N}$ and P205 for better growth and flower production of dahlia cv. "Kenya yellow". Kadu et al., (2009) reported that the effect of nitrogen, phosphorus and potassium on growth, flowering and bulb production in tuberose cv. Single, an experiments were conducted in two season to study the effect of four levels, each of nitrogen $(0,100,200$ and $300 \mathrm{~kg} / \mathrm{ha})$ and phosphorus $(0.100 .200$ and $300 \mathrm{~kg} / \mathrm{ha}-1)$ with a fixed levels of potassium @ 100kg/ha-1 in tuberose. Kazemi et al., (2011) reported that several documents based on using different treatments in postharvest for delaying senescence and enhancing cut flower vase life such as Eustoma grandiflorum. Kishore et al., (2010) reported that the effect of different levels of nitrogen, phosphorus and potassium on growth and flowering of African marigold cv. Pusha Narangi. Khalighi et al., (2007) reported that the effects of $\mathrm{N}, \mathrm{P}$ and $\mathrm{K}$ fertilizer ratio on the bulb quality and yield of tulip. Nair et al., (2000) reported that the P. tuberosa cv. Double plants were treated with varying NPK rates $(15: 30: 15,30: 30: 15$, $15: 60: 15,30: 60 ; 15,15: 90: 15.30: 90$ : $15 \mathrm{~g} / \mathrm{m} 2) 1997-98$ to standardize the $\mathrm{P}$ and $\mathrm{N}$ requirements for the optimum growth and yield of flowers and bulbs of tuberose. . Jamshidi et al., (2012) reported that that a flower's vase life when held in a solution containing $1 \mathrm{mM} \mathrm{SA}$ and $2 \mathrm{mM}$ MA was significantly higher than that of the control treatments. 
Memon et al., (2013) concluded that potassium nitrate solution upto $3 \%$ concentration as presoaking treatment and foliar application on gladiolus plants positively influenced almost all the parameters including extended vase life of out flowers. Niedziela et al., (2008) reported that effect of N.P and K resulted in stem bulb fresh weight, stem plus leaf fresh weight, number of flower and stem root fresh weight, And only when all three nutrients were omitted in lily plant. Singh and Kumar (2010) studied the effect of nitrogen with combination of phosphorus and potassium on growth, flower yield and quality of four indigenous cut flower rose varieties namely Pusa Gaurav. Growth and flower yield was increased significantly with application of optimum level of nitrogen in combination with phosphorus and potassium $(9 \mathrm{~g} \mathrm{~N}+8 \mathrm{~g}$ $\mathrm{P}_{2} \mathrm{O}_{5}+8 \quad \mathrm{~g} \quad \mathrm{~K}_{2} \mathrm{O} / \mathrm{m}^{2} /$ week) through top dressings $(1,100,200$ and $300 \mathrm{~kg} / \mathrm{ha})$ and recorded highest plant height. Singh et al., (2008) reported that effect of four level each of nitrogen $(100.15030$ and $250 \mathrm{~kg} / \mathrm{ha})$ and potassium $(120,180,240$ and $300 \mathrm{~kg} / \mathrm{ha})$ with a fixed level of phosphorus@50kg/'ha in Asiatic hybrid lily, the NPK combination treatment of $250+50+300 \mathrm{~kg} / \mathrm{ha}$. Respectively showed highest length and diameter of flowering shoot, number of leaves per shoot, diameter and of flower buds per shoot. As the combination of NPK treatment were given 45 days after planting of bulbs. There was significant effect on plant height, it is well known that chemical fertilizer could enhance plant growth due to the role of nitrogen in nucleic acid and protein synthesis and phosphorus as an essential component of the energy compounds (ATP and ADP) and potassium as an activator of many enzymes (Helgi and Rolfe, 2005) in the apical meristem plants have meristic tissue in several location, both roots and shoots, cell division and cell elongation in the apical meristem is called primary growth and result in an increase in plant height. The combination of NPK increase intensity of cell division and cell elongation, Increasing shoot length makes the plant taller, thus allowing it better access to sunlight for photosynthesis.

In Polyhouse maximum plant height (34.29 $\mathrm{cm})$ at $60 \mathrm{DAP}$, was recorded with treatment $\mathrm{T}_{2}$ (Foliar application of NPK 10:10:10 @ $5 \mathrm{~g} / \mathrm{l}$ thrice a week) closely followed by $(33.91 \mathrm{~cm}) \mathrm{T}_{3}$ (Foliar application of NPK 10:10:10 @ 5g/l twice a week) and the minimum $(27.84 \mathrm{~cm})$ was recorded with $\mathrm{T}_{1}$ (Control (FYM mixed to the soil @ 1m ${ }^{3}$ per $100 \mathrm{~m}^{2}$ area). In open condition maximum plant height $(12.21 \mathrm{~cm})$ at $30 \mathrm{DAP}$, was recorded with treatment $\mathrm{T}_{2}$ (Foliar application of NPK 10:10:10 @ 5g/l thrice a week) closely followed by $(8.85 \mathrm{~cm}) \mathrm{T}_{3}$ (Foliar application of NPK 10:10:10 @ 5g/l twice a week) and the minimum $(5.31 \mathrm{~cm})$ was recorded with $\mathrm{T}_{1}$ (Control (FYM mixed to the soil @ $1 \mathrm{~m}^{3}$ per $100 \mathrm{~m}^{2}$ area).

In open condition maximum plant height $(22.15 \mathrm{~cm})$ at $60 \mathrm{DAP}$, was recorded with treatment $\mathrm{T}_{2}$ (Foliar application of NPK 10:10:10 @ 5g/l thrice a week) closely followed by $(19.86 \mathrm{~cm}) \mathrm{T} 3$ (Foliar application of NPK 10:10:10 @ 5g/l twice a week) and the minimum $(16.18 \mathrm{~cm})$ was recorded with $\mathrm{T}_{1}$ (Control (FYM mixed to the soil @ 1m ${ }^{3}$ per $100 \mathrm{~m}^{2}$ area).

Polyhouse was found to be better for plant growth (height) of Lilium under of Odisha conditions

\section{Flowering characters}

A perusal of data in Table $b$ shows that maximum flowering. In polyhouse flower length $(139.30 \mathrm{~mm})$, flower diameter $(133.62 \mathrm{~mm})$, bud length $(106.21 \mathrm{~mm})$, bud diameter $(5.11 \mathrm{~mm})$, first flower senescence (14.67 days), number of bud per plant (3.66), 
vase life of Lilium (14.67 days), of Lilium was maximum in the treatment $\mathrm{T}_{2}$ (Foliar application of NPK 10:10:10 @ 5g/l thrice a week).In open condition flower length $(145.57 \mathrm{~mm})$ flower diameter(141.41 mm), bud length $(17.45 \mathrm{~mm})$, bud diameter $(2.67 \mathrm{~mm})$, days to bud emergence (38.36 days), first flower senescence (10.00 days), number of bud per plant (2.33), vase life (10.00 days), of Lilium was maximum in the treatment $\mathrm{T}_{2}$ (Foliar application of NPK 10:10:10 @ 5g/1 thrice a week) Foliar application of NPK must have resulted in higher translocation of carbohydrates from vegetative parts to the reproductive parts which quality may have resulted in better development of flower size. Followed by treatment $\mathrm{T}_{3}$ (Foliar application of NPK 10:10:10@5g/l twice a week) (129.57 mm) and the minimum $(120.43 \mathrm{~mm})$ was recorded with $\mathrm{T}_{1}$ (Control (FYM mixed to the soil @ $1 \mathrm{cum}$ per $100 \mathrm{~m}^{2}$ area) Similar trend was observed at subsequent growth stages also.

As the combination of NPK treatment was given to the planting of bulbs. There was significant effect on spike length due to the role of nitrogen in nucleic acid and protein synthesis and phosphorus as an essential component of the energy compounds (ATP and ADP) and potassium as an activator of many enzymes (Helgi and Rolfe, 2005)

Table.1(A) Effect of foliar application and fertigation of NPK on Lilium and comparison between polyhouse P1 and open condition P2 (Growth Characters)

\begin{tabular}{|c|c|c|c|c|c|c|c|c|c|c|c|}
\hline \multicolumn{2}{|c|}{ Treatments } & \multicolumn{5}{|c|}{ Polyhouse (P1) } & \multicolumn{5}{|c|}{ Open Condition (P2) } \\
\hline & & $\begin{array}{l}\text { Plant } \\
\text { height }\end{array}$ & $\begin{array}{l}\text { No. of } \\
\text { leaves/ } \\
\text { plant }\end{array}$ & $\begin{array}{l}\text { Plant } \\
\text { spread }\end{array}$ & $\begin{array}{l}\text { Spike } \\
\text { length }\end{array}$ & $\begin{array}{l}\text { Spike } \\
\text { diameter }\end{array}$ & $\begin{array}{l}\text { Plant } \\
\text { height }\end{array}$ & $\begin{array}{l}\text { No. of } \\
\text { leaves/p } \\
\text { lant }\end{array}$ & $\begin{array}{l}\text { Plant } \\
\text { spread }\end{array}$ & $\begin{array}{l}\text { Spike } \\
\text { length }\end{array}$ & $\begin{array}{l}\text { Spike } \\
\text { diameter }\end{array}$ \\
\hline \multirow[t]{2}{*}{$\mathrm{T}_{1}$} & \multirow{2}{*}{$\begin{array}{l}\text { Control (FYM mixed to } \\
\text { the soil @ } 1 \mathrm{~m}^{3} \text { per } 100 \mathrm{~m}^{2} \\
\text { area) }\end{array}$} & $\begin{array}{l}60 \\
\text { DAP }\end{array}$ & $\begin{array}{l}60 \\
\text { DAP }\end{array}$ & $\begin{array}{l}60 \\
\text { DAP }\end{array}$ & $\begin{array}{l}60 \\
\text { DAP }\end{array}$ & $\begin{array}{l}60 \\
\text { DAP }\end{array}$ & $\begin{array}{l}60 \\
\text { DAP }\end{array}$ & $\begin{array}{l}60 \\
\text { DAP }\end{array}$ & $\begin{array}{l}60 \\
\text { DAP }\end{array}$ & $\begin{array}{l}60 \\
\text { DAP }\end{array}$ & $\begin{array}{l}60 \\
\text { DAP }\end{array}$ \\
\hline & & 27.84 & 49.27 & 12.45 & 35.75 & 4.33 & 16.18 & 31.15 & 7.00 & 22.58 & 3.54 \\
\hline $\mathbf{T 2}$ & $\begin{array}{l}\text { Foliar application of NPK } \\
\text { 10:10:10@ } 9 \mathrm{~g} / 1 \text { thrice a } \\
\text { week }\end{array}$ & 34.29 & 64.68 & 14.67 & 39.73 & 4.95 & 22.15 & 55.36 & 12.68 & 29.98 & 4.14 \\
\hline $\mathrm{T}_{3}$ & $\begin{array}{l}\text { Foliar application of NPK } \\
\text { 10:10:10 @ 5g/l twice a } \\
\text { week }\end{array}$ & 33.91 & 60.65 & 14.00 & 38.96 & 4.88 & 19.86 & 41.20 & 12.00 & 27.98 & 4.04 \\
\hline $\mathrm{T}_{4}$ & $\begin{array}{l}\text { (Foliar application of } \\
\text { NPK 10:10:10 @ 5g/1 } \\
\text { once a week) }\end{array}$ & 29.82 & 54.68 & 12.97 & 38.44 & 4.56 & 17.25 & 33.36 & 8.16 & 24.94 & 3.86 \\
\hline $\mathrm{T}_{5}$ & $\begin{array}{l}\text { Soil drenching with NPK } \\
\text { 10:10:10@ } 5 \mathrm{~g} / 1 \text { thrice a } \\
\text { week }\end{array}$ & 32.31 & 57.67 & 13.21 & 38.83 & 4.85 & 18.36 & 40.15 & 9.15 & 27.84 & 4.03 \\
\hline $\mathrm{T}_{6}$ & $\begin{array}{l}\text { Soil drenching with NPK } \\
\text { 10:10:10@5g/l twice a } \\
\text { week }\end{array}$ & 30.77 & 55.69 & 13.03 & 38.66 & 4.69 & 18.15 & 35.87 & 8.36 & 26.37 & 4.02 \\
\hline \multirow[t]{4}{*}{$\mathrm{T}_{7}$} & $\begin{array}{l}\text { Soil drenching with NPK } \\
\text { 10:10:10@5g/l once a } \\
\text { week }\end{array}$ & 28.92 & 51.15 & 12.57 & 36.86 & 4.50 & 18.15 & 32.00 & 7.21 & 23.29 & 3.74 \\
\hline & F- test & $\mathrm{S}$ & $\mathrm{S}$ & $\mathrm{S}$ & $\mathrm{S}$ & $\mathrm{S}$ & $\mathrm{S}$ & $\mathrm{S}$ & $\mathrm{S}$ & $\mathrm{S}$ & $\mathrm{S}$ \\
\hline & S. Ed. $( \pm)$ & 0.486 & 0.746 & 0.614 & 0.971 & 0.147 & 0.542 & 0.626 & 0.688 & 2.360 & 0.409 \\
\hline & C. D. $(P=0.05)$ & 1.003 & 1.540 & 1.267 & 2.004 & 0.303 & 1.118 & 1.292 & 1.420 & 4.872 & 0.844 \\
\hline
\end{tabular}


Table.1(B.) Effect of foliar application and fertigation of NPK on Lilium and comparison between polyhouse P1 and open condition P2 (flowering Characters)

\begin{tabular}{|c|c|c|c|c|c|c|c|c|c|}
\hline & \multirow[t]{3}{*}{ Treatments } & \multicolumn{8}{|c|}{ Polyhouse (P1) } \\
\hline & & $\begin{array}{l}\vec{E} \\
000 \\
\overline{0} \\
\overline{\bar{d}} \\
\overrightarrow{0} \\
\frac{0}{I}\end{array}$ & 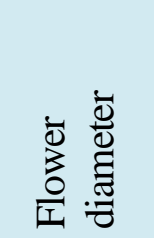 & $\begin{array}{l}\frac{\vec{Z}}{00} \\
\overline{0} \\
\frac{0}{0} \\
\overline{0}\end{array}$ & 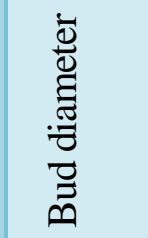 & 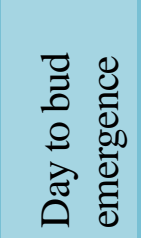 & 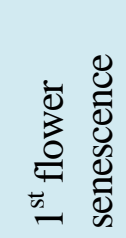 & 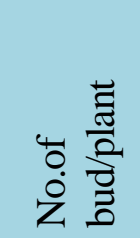 & 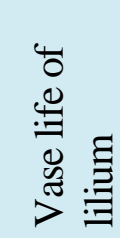 \\
\hline & & $\begin{array}{l}60 \\
\text { DAP }\end{array}$ & 60 DAP & 60DAP & 60DAP & $\begin{array}{l}60 \\
\text { DAP }\end{array}$ & $\begin{array}{l}60 \\
\text { DAP }\end{array}$ & $\begin{array}{l}60 \\
\text { DAP }\end{array}$ & $\begin{array}{l}60 \\
\text { DAP }\end{array}$ \\
\hline $\mathrm{T}_{1}$ & $\begin{array}{l}\text { Control (FYM mixed to } \\
\text { the soil @ } 1 \mathrm{~m}^{3} \text { per } 100 \\
\mathrm{~m}^{2} \text { area) }\end{array}$ & 120.43 & 121.53 & 101.86 & 4.42 & 39.35 & 12.77 & 2.77 & 12.77 \\
\hline $\mathrm{T} 2$ & $\begin{array}{l}\text { Foliar application of } \\
\text { NPK 10:10:10 @ 5g/l } \\
\text { thrice a week }\end{array}$ & 139.30 & 133.62 & 106.21 & 5.11 & 48.33 & 14.67 & 3.66 & 14.67 \\
\hline $\mathrm{T}_{3}$ & $\begin{array}{l}\text { Foliar application of } \\
\text { NPK 10:10:10 @ 5g/1 } \\
\text { twice a week }\end{array}$ & 129.57 & 132.25 & 105.06 & 4.89 & 47.33 & 14.22 & 3.33 & 14.22 \\
\hline $\mathrm{T}_{4}$ & $\begin{array}{l}\text { (Foliar application of } \\
\text { NPK 10:10:10 @ 5g/l } \\
\text { once a week) }\end{array}$ & 125.36 & 127.16 & 103.60 & 4.78 & 40.21 & 13.22 & 2.98 & 13.22 \\
\hline $\mathrm{T}_{5}$ & $\begin{array}{l}\text { Soil drenching with NPK } \\
\text { 10:10:10@ } 9 \mathrm{~g} / 1 \text { thrice a } \\
\text { week }\end{array}$ & 129.14 & 131.79 & 104.87 & 4.89 & 43.66 & 13.44 & 3.22 & 13.44 \\
\hline $\mathrm{T}_{6}$ & $\begin{array}{l}\text { Soil drenching with NPK } \\
\text { 10:10:10 @ 5g/l twice a } \\
\text { week }\end{array}$ & 128.31 & 128.47 & 104.22 & 4.78 & 43.15 & 13.22 & 3.11 & 13.22 \\
\hline \multirow[t]{4}{*}{$\mathrm{T}_{7}$} & $\begin{array}{l}\text { Soil drenching with NPK } \\
\text { 10:10:10 @ } 5 \mathrm{~g} / 1 \text { once a } \\
\text { week }\end{array}$ & 123.95 & 126.05 & 102.54 & 4.55 & 40.00 & 13.00 & 2.78 & 13.00 \\
\hline & F- test & $S$ & $\mathrm{~S}$ & NS & $\mathrm{S}$ & $\mathrm{S}$ & $S$ & $\mathrm{~S}$ & $S$ \\
\hline & S. Ed. $( \pm)$ & 4.812 & 3.101 & 3.415 & 0.177 & 0.725 & 0.541 & 0.209 & 0.541 \\
\hline & C. D. $(P=0.05)$ & 9.932 & 6.401 & 7.048 & 0.366 & 1.497 & 1.117 & 0.431 & 1.117 \\
\hline
\end{tabular}


Table.2 Effect of foliar application and fertigation of NPK on Lilium and comparison between polyhouse P1 and open condition P2 (flowering Characters)

\begin{tabular}{|c|c|c|c|c|c|c|c|c|c|}
\hline \multicolumn{2}{|r|}{ Treatments } & \multicolumn{8}{|c|}{ Open Condition (P2) } \\
\hline & & \multirow{2}{*}{ 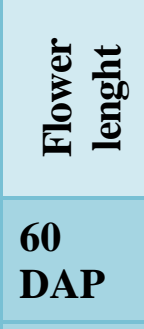 } & \multirow{2}{*}{ 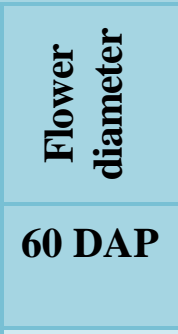 } & \multirow{2}{*}{ 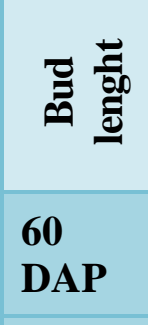 } & \multirow{2}{*}{ 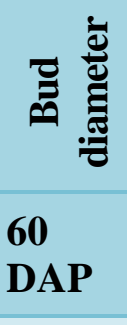 } & \multirow{2}{*}{$\begin{array}{l}\text { Q } \\
\text { D0 } \\
\text { DAP }\end{array}$} & \multirow{2}{*}{ 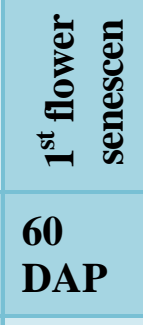 } & \multirow{2}{*}{ 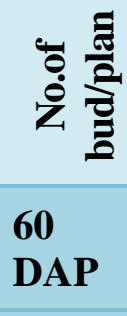 } & \multirow{2}{*}{ 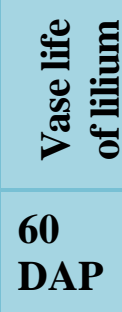 } \\
\hline & & & & & & & & & \\
\hline $\mathrm{T}_{1}$ & $\begin{array}{l}\text { Control (FYM mixed to } \\
\text { the soil @ } 1 \mathrm{~m}^{3} \text { per } 100 \\
\mathrm{~m}^{2} \text { area) }\end{array}$ & 137.31 & 121.57 & 12.06 & 1.33 & 30.18 & 6.67 & 1.00 & 6.67 \\
\hline $\mathrm{T} 2$ & $\begin{array}{l}\text { Foliar application of } \\
\text { NPK 10:10:10@ } 5 \mathrm{~g} / 1 \\
\text { thrice a week }\end{array}$ & 145.57 & 141.41 & 17.45 & 2.67 & 38.36 & 10.00 & 2.33 & 10.00 \\
\hline $\mathrm{T}_{3}$ & $\begin{array}{l}\text { Foliar application of } \\
\text { NPK 10:10:10 @ 5g/l } \\
\text { twice a week }\end{array}$ & 144.46 & 135.36 & 16.51 & 2.67 & 36.15 & 9.79 & 2.33 & 9.79 \\
\hline $\mathrm{T}_{4}$ & $\begin{array}{l}\text { (Foliar application of } \\
\text { NPK 10:10:10 @ 5g/l } \\
\text { once a week) }\end{array}$ & 142.22 & 126.26 & 14.34 & 2.33 & 32.36 & 9.26 & 1.33 & 9.26 \\
\hline $\mathrm{T}_{5}$ & $\begin{array}{l}\text { Soil drenching with } \\
\text { NPK 10:10:10@ } @ \mathrm{~g} / 1 \\
\text { thrice a week }\end{array}$ & 143.96 & 132.00 & 15.47 & 2.67 & 35.15 & 9.46 & 1.67 & 9.46 \\
\hline $\mathrm{T}_{6}$ & $\begin{array}{l}\text { Soil drenching with } \\
\text { NPK 10:10:10 @ } \\
\text { 5g/l twice a week }\end{array}$ & 143.45 & 129.19 & 14.56 & 2.33 & 35.00 & 9.45 & 1.67 & 9.45 \\
\hline $\mathrm{T}_{7}$ & $\begin{array}{l}\text { Soil drenching with } \\
\text { NPK 10:10:10 @ } \\
\text { 5g/l once a week }\end{array}$ & 139.08 & 125.23 & 13.84 & 1.33 & 30.21 & 6.96 & 1.33 & 6.96 \\
\hline & F- test & $S$ & $\mathrm{~S}$ & $S$ & $S$ & $S$ & $S$ & $\mathrm{~S}$ & $\mathrm{~S}$ \\
\hline & S. Ed. $( \pm)$ & 1.951 & 4.026 & 1.974 & 0.437 & 0.577 & 0.945 & 0.393 & 0.945 \\
\hline & C. D. $(P=0.05)$ & 4.028 & 8.309 & 4.074 & 0.903 & 1.192 & 1.950 & 0.811 & 1.950 \\
\hline
\end{tabular}


Table.3 Cost of cultivation for Lilium (Asiatic lily) hybrid Tresor fixed cost for all treatments

\begin{tabular}{|c|c|c|c|c|c|}
\hline SI.NO. & Particulars & Unit & Qty. & Rate/Unit(Rs.) & $\operatorname{Cost}($ Rs./ha) \\
\hline & $\begin{array}{l}\text { Land Preparation } \\
\text { Ploughing } \\
\text { Disc harrowing } \\
\text { Levelling with Cultivar } \\
\text { Layout of the field }\end{array}$ & $\begin{array}{l}\text { Hours } \\
\text { Hours } \\
\text { Hours } \\
\text { Labour }\end{array}$ & $\begin{array}{l}3 \\
3 \\
3 \\
6\end{array}$ & $\begin{array}{l}500 \\
500 \\
500 \\
160\end{array}$ & $\begin{array}{l}1,500 \\
1500 \\
1500 \\
960\end{array}$ \\
\hline B. & $\begin{array}{l}\text { Cuttings and Transplanting } \\
\text { 1. Cost of Bulbs } \\
\text { 2. Labour for planting }\end{array}$ & $\begin{array}{l}\text { Nos. } \\
\text { Labour }\end{array}$ & $\begin{array}{l}40000 \\
20\end{array}$ & $\begin{array}{l}13 \\
160\end{array}$ & $\begin{array}{l}5200,00 \\
3,200\end{array}$ \\
\hline C. & $\begin{array}{l}\text { Fertilizer application + } \\
\text { Irrigation } \\
\text { Foliar application and } \\
\text { fertigation weekly } \\
\text { Labour for application of } \\
\text { fertilizer } \\
\text { Tubewell Charges } \\
\text { Labour per irrigation }\end{array}$ & Labour & $\begin{array}{l}25 \\
4 \\
8\end{array}$ & $\begin{array}{l}160 \\
400 \\
160\end{array}$ & $\begin{array}{l}4,000 \\
1600 \\
1,280\end{array}$ \\
\hline D. & $\begin{array}{l}\text { Inter-culture } \\
\text { 1.Thinningb and Gap Filling } \\
\text { 2.4 Hand } \\
\text { Weeding(2labours/weeding) }\end{array}$ & $\begin{array}{l}\text { Labour } \\
\text { Labour }\end{array}$ & $\begin{array}{l}4 \\
8\end{array}$ & $\begin{array}{l}160 \\
160\end{array}$ & $\begin{array}{l}640 \\
1,280\end{array}$ \\
\hline F. & $\begin{array}{l}\text { Plant protection measures } \\
\text { 1. Cost of insecticide } \\
\text { 2. Applicationing of } \\
\text { Insecticides }\end{array}$ & $\begin{array}{l}\mathrm{Kg} \\
\text { Labour }\end{array}$ & $\begin{array}{l}4 \\
8\end{array}$ & $\begin{array}{l}300 \\
160\end{array}$ & $\begin{array}{l}1,200 \\
1,280\end{array}$ \\
\hline G. & $\begin{array}{l}\text { Harvesting } \\
\text { 1. Labour for harvesting and } \\
\text { packing } \\
\text { 2. transportation }\end{array}$ & Labour & 40 & 160 & $\begin{array}{l}6,400 \\
4,000\end{array}$ \\
\hline H. & Rental value of land & Months & 8 & 500 & 4,000 \\
\hline \multirow[t]{2}{*}{ I. } & Supervision charges & Month & 8 & 3000 & 24,000 \\
\hline & Total & & & & 5,78340 \\
\hline
\end{tabular}


Table.4 Economics of different treatments and benefit cost: ratio for cultivation of Lilium (Asiatic lily) hybrid Tresor

\begin{tabular}{|c|c|c|c|c|c|c|c|}
\hline & 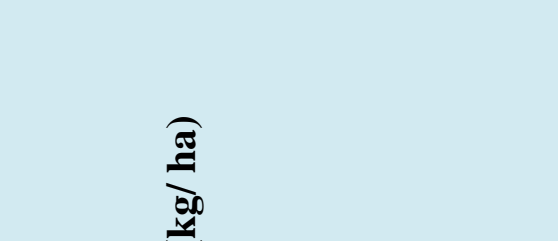 & & 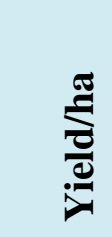 & 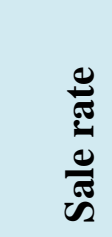 & 党: & 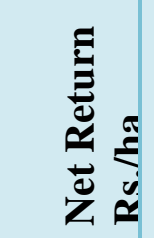 & 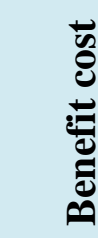 \\
\hline 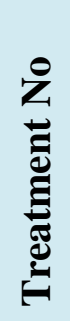 & 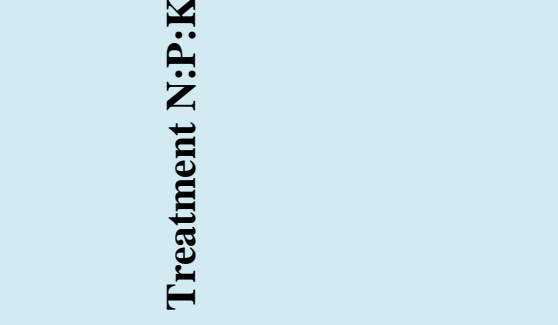 & 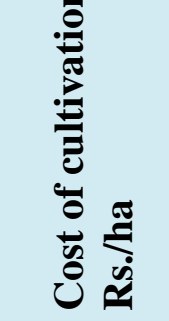 & 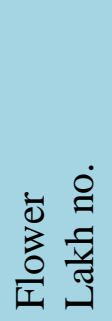 & $\frac{\dot{0}}{\dot{0}} \dot{\Delta}$ & & & \\
\hline T1 & $\begin{array}{l}\text { (Control (FYM mixed to the soil } \\
\text { @ } 1 \mathrm{~m}^{3} \text { per } 100 \mathrm{~m}^{2} \text { area) }\end{array}$ & 5,70460 & 0.40 & 25 & $\begin{array}{c}10,00 \\
000\end{array}$ & $\begin{array}{c}42954 \\
0\end{array}$ & 1.75 \\
\hline T2 & $\begin{array}{c}\text { (Foliar application of NPK } \\
\text { 10:10:10@ } 9 \mathrm{~g} / 1 \text { thrice a week) }\end{array}$ & 5,78590 & 0.65 & 25 & $\begin{array}{c}16,25 \\
000\end{array}$ & $\begin{array}{c}10464 \\
10\end{array}$ & 2.80 \\
\hline T3 & $\begin{array}{c}\text { (Foliar application of NPK } \\
\text { 10:10:10 @ 5g/l twice a week) }\end{array}$ & 5,75340 & 0.62 & 25 & $\begin{array}{c}15,50 \\
000\end{array}$ & $\begin{array}{c}97466 \\
0\end{array}$ & 2.69 \\
\hline T4 & $\begin{array}{c}\text { (Foliar application of NPK } \\
\text { 10:10:10@ 5g/l once a week) }\end{array}$ & 5,71340 & 0.58 & 25 & $\begin{array}{c}14,50 \\
000\end{array}$ & $\begin{array}{c}87866 \\
0\end{array}$ & 2.53 \\
\hline T5 & $\begin{array}{c}\text { (Soil drenching with NPK } \\
\text { 10:10:10@ } 5 \mathrm{~g} / 1 \text { thrice a week ) }\end{array}$ & 5,73220 & 0.63 & 25 & $\begin{array}{c}15,75 \\
000\end{array}$ & $\begin{array}{c}10017 \\
80\end{array}$ & 2.74 \\
\hline T6 & $\begin{array}{c}\text { (Soil drenching with NPK } \\
\text { 10:10:10 @ 5g/l twice a week) }\end{array}$ & 5,72460 & 0.59 & 25 & $\begin{array}{c}14,75 \\
000\end{array}$ & $\begin{array}{c}90254 \\
0\end{array}$ & 2.57 \\
\hline T7 & $\begin{array}{c}\text { (Soil drenching with NPK } \\
\text { 10:10:10 @ 5g/l once a week) }\end{array}$ & 5,71340 & 0.55 & 25 & $\begin{array}{c}13,75 \\
000\end{array}$ & $\begin{array}{c}80366 \\
0\end{array}$ & 2.40 \\
\hline
\end{tabular}

Fig.1 Effect of foliar application and fertigation of NPK on Lilium and comparison between polyhouse $\mathrm{P} 1$ and open condition $\mathrm{P} 2$

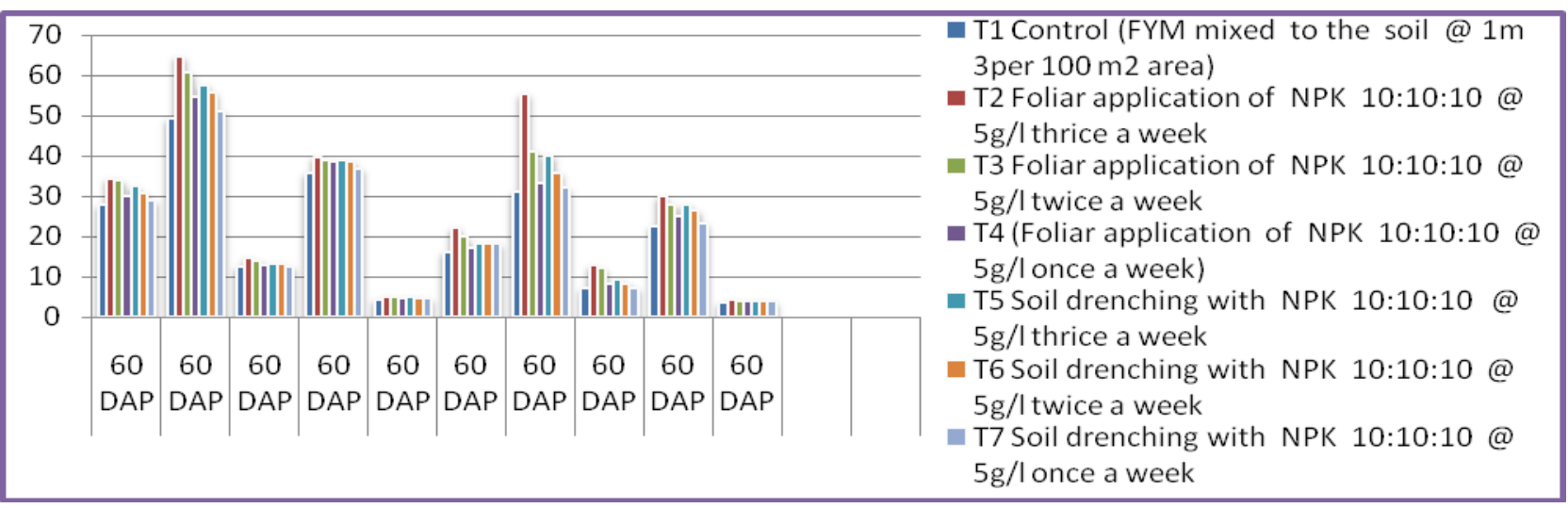


Fig.2 Effect of foliar application and fertigation of NPK on Lilium polyhouse P1

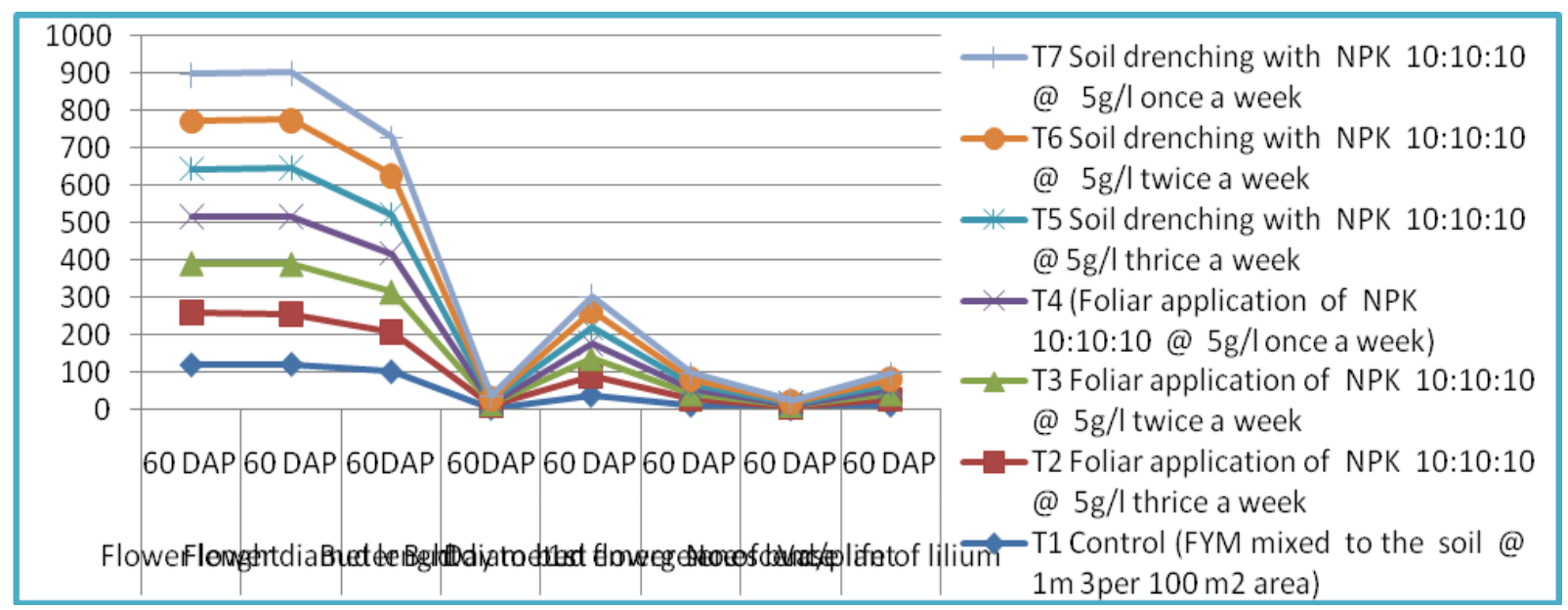

Fig.3 Effect of foliar application and fertigation of NPK on Lilium open condition P2

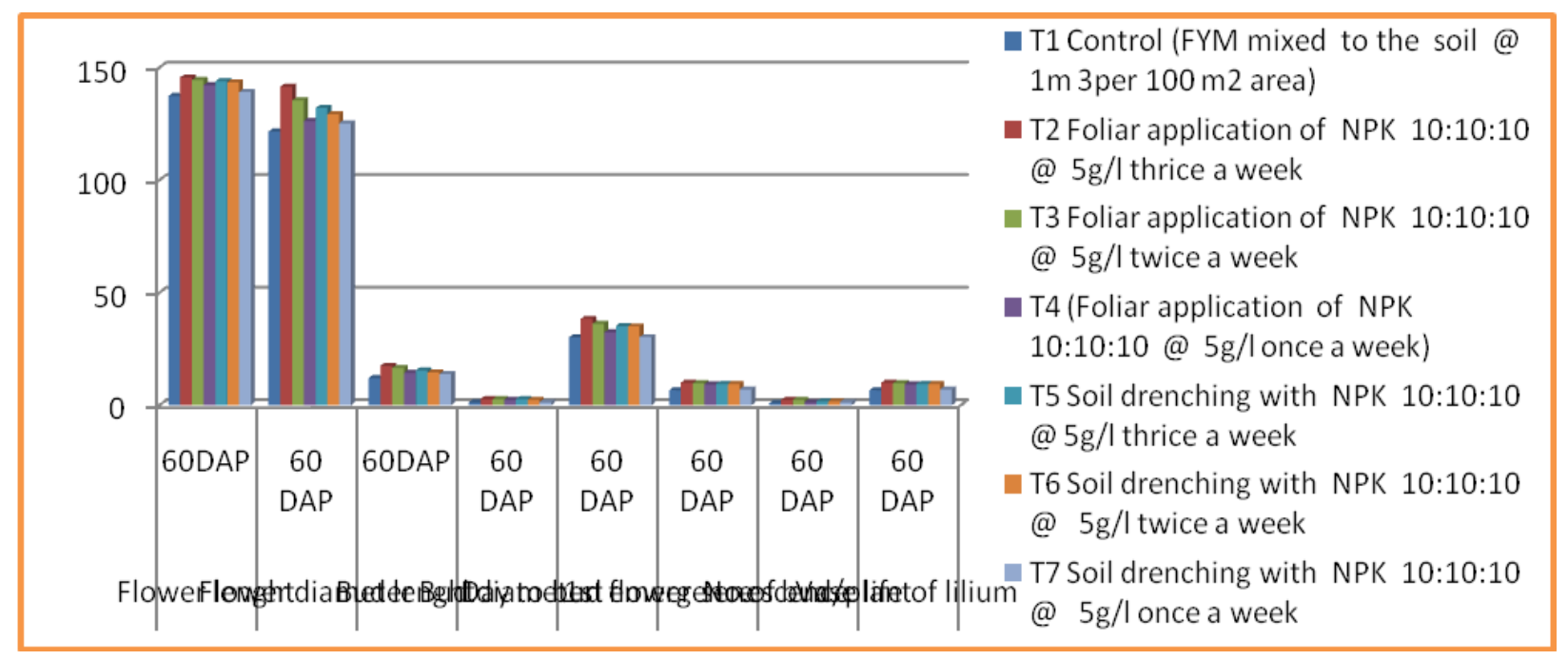

Polyhouse was found to be better for flower quality (flower length) of Lilium under Odisha conditions. The results of the study revealed that application of T2 (Foliar application of NPK 10:10:10 @ 5g/l thrice a week) recorded significantly higher.

\section{Economics of different treatments}

Maximum gross return was recorded in treatment $\mathrm{T}_{2}$ with (Foliar application of NPK 10:10:10@5g/l thrice a week) (Rs1625000 /ha) followed by $\mathrm{T}_{3}$ (Foliar application of
NPK 10:10:10 @ 5g/l twice a week) $(15,50000 / \mathrm{ha})$ and the minimum (Rs.1000000/ha) was recorded in treatment $\mathrm{T}_{1}$ (Control (FYM mixed to the soil @ $1 \mathrm{~m}^{3}$ per $100 \mathrm{~m}^{2}$ area).

Maximum net return was recorded in treatment $\mathrm{T}_{2}$ with (Foliar application of NPK 10:10:10 @ 5g/l thrice a week) (Rs1046660/ha) and the minimum (Rs429540/ha) was recorded in treatment $\mathrm{T}_{1}$ (Control (FYM mixed to the soil @ $1 \mathrm{~m}^{3}$ per $100 \mathrm{~m}^{2}$ area). 
Maximum Benefit cost ratio was recorded in $\mathrm{T}_{2}$ with (Foliar application of NPK 10:10:10 @ 5g/l thrice a week) (2.80) and the minimum (1.75) was recorded in treatment $\mathrm{T}_{1}$ (Control (FYM mixed to the soil @ $1 \mathrm{~m}^{3}$ per $100 \mathrm{~m}^{2}$ area)

As the economics is the need of the farmers while taking decision regarding the adoption of the technique and scientific knowledge Hence, NPK gave the highest gross return, net return, and benefit: cost ratio was due to higher productivity and higher quality of flowers, which increase the market value of the flowers.

In conclusion, polyhouse was found to be better for flower quality (flower length) of Lilium under Odisha conditions. The results of the study it could be inferred that the effect of Foliar application of NPK 10:10:10 @ 5g/1 thrice a week on growth, flowering lilium hybrid showed best response on applying Foliar application of NPK 10:10:10 @ 5g/l thrice a week foliar spray on plants, thus it could be concluded that the plants of lilium hybrid will show best growth, flowering and yield response under Polyhouse. Foliar application of NPK 10:10:10 @ 5g/l thrice a week foliar spray.

\section{References}

Acharya, M. M. and Dashora, L.K. (2004). Response of graded levels of nitrogen and phosphorus on vegetative growth and flowering in African marigold (Tagetes erecta L.) I Orna. Hort. 7: (2) 179-183.

Arya, G. R. J. K. and Gbalot, P. K. (2010). Effect of different levels of nitrogen, phosphorus and potassium on growth and flowering of African marigold cv. Pusa Narangi. Prog. Agri. 10(1): 8- 83.

Baloch, Q.B., Chachar, Q.I. and Panhwar, U.I. (2010).Effect of NP fertilizers on the growth and flower production of Zinnia (Zinnia elegans L). J. Agri. Tech 6: (1)193-200.

Bhat, Z. A. and Shepherd, H. (2006). Effect of source and level of nitrogen on growth, flowering and yield in African marigold (Tagetes erecta L.) J. Orna. Hort. 9:(3)218-220.

Birade, R. M. Ranpise,S. A. Patil, B. T. and Ghure, T. K. (2003). Effect of NPK and spacing's on growth, quality and flower production in China Aster (Callistephus chinensis L.) Advance in Hort. and Forestry. 9: (2) 243-249.

Beura, D. and Singh, A. (2002). Effect of integrated nutrient management on Vegetative growth and flowering attributes of tuberose (Polianthes tuberosa Linn) cv. Hyderabad Double. Res. Environ. Life 8 (1) 15-17.

Bankar, R A. Biradar, S.L and Bhakuni DS, Jain S (1990). Chemistry of cultivated medicinal plants: Gloriosa superba Linn. (Agnishikha, Liliaceae). In: Chadha KL, Gupta Rajendra (eds) Advances in Horticulture Vol. 11, Malhotra Publishing House, Delhi. pp. 98-99.

Chaturvedi, R.K. and Misra, R. L. (2003). Effect of $\mathrm{N}$ and $\mathrm{P}$ on growth and flowering of gladiolus. J. Hon. Orna. 6(2): 100-103.

Dole J., Wilkins H. (1996). Direction of Lilium research. Acta Hortic. 414, 295300

Dole J., Wilkins H. (1999). Lilium, Asiatic and Oriental, Floriculture Principles and Species. Upper Saddle River, NJ: Prentice Hall, 408-416

Devi Khwairakpam Lily, and Singh U.C. (2010). Effect of nitrogen on growth, flowering and yield of tuberose (Polianthes tuberosa L.) cv. Single. J. Orna- Hon. 13(3): 228-232.

Grassotti, S. M. Z. Oraghi Ardebili. and Din, A. (2011). 'Growth and blossoming of 
Lilium under various organic fertilizers. Iranian Journal of Plant Physiology 5 (1), 1235-1242.

Gani, G. Beigh, M. A. Lone, R. A. Nanda, A. B. and Hussain, K. (2007). Effect of different levels of nitrogen and phosphorus on growth and flowering of dahlia cv. Kenya yellow. J. Plan! Set Res. 23: (1/2) 59-62.

Ghule, A. D. Patil, P. V. and Kantharaju, K. T. (2003). Effect of different levels of nitrogen and phosphorus on growth and flowering of spider lily. J. Maharashtra Agri. Univ.28: (2) 128-130.

Helgl, S and Rolfe M. N. (2005) Growth and blossoming of Lilium under various organic fertilizers '. Iranian Journal of Plant Physiology 5 (1), 1235-1242.

Jamshidi M., Hadavi E. and Naderi R (2012). Effects of Salicylic Acid and Malic Acid on vase life and bacterial and yeast populations of preservative solution in cut Gerbera flowers. International Journal of Agri Science. 2(8): 671-674

Kazemi M., Hajizadeh H. S., Gholami M., Asadi M., Aghdasi S. (2011). Efficiency of Essential Oils, Citric Acid, Malic Acid And Nickel Reduced Ethylene Production And Extended Vase Life of Cut Lisianthus Flowers. Research Journal of Botany. 7:14-18

Kishore, G.R. and Singh, P.V. (2010).: Effect of $\mathrm{N}, \mathrm{P}$ or $\mathrm{K}$ fertilizers on vegetative growth of tuberose (Polianthes tuberosa L.) cv. Single,. Plant Archives, 6: 377 38

Kadu, A. P. Kadu, P.R. and Sable, A.S. (2009). Effect of nitrogen, phosphorus and potassium on growth, flowering and bulb production in tuberose cv. Single. $J$ Soils and Crops 19: (2)367-370.

Khalighi, A. Hojjati, Y. Babalar, ML and Nadari, R. (2007). Effect of fertilizer ratio $\mathrm{N} \mathrm{P} K$ on bulb quality, and quantity characteristics of tulip cv: hybrid Darwin Apledoorn. [Persian]
IranianJ. AgriSci. 38:1 39-45.

Khan, F. U. Jhon, A. Q. Khan, F. A. and Mir, M. M. (2006). Effect of NPK and Zn on growth, flowering and bulb production in tulip under polyhouse conditions in Kashmir. J. Hort. Sci. 1:(2) 129-134.

Kumar Neeraj Rani Ramesh and Dhatt, K. K. (2005). Effect of nitrogen levels and growing media on growth, flowering and bulb production of Lilium cultivars. J.Orna. Hort. (New Series). 8: (1) 3640.

Kumar Ratan and S. S. Singh. (2009). Effect of nitrogen and phosphorus on growth, flowering and bulb production in Asiatic hybrid Lily cv. Novecento. J. Orna.Hort. 12 (2):142-145.

Singh, M. K. and S. Kumar (2010). Effect of NPK on growth and flowering of oil flower Rose (Rosa hybrida) under polyhouse conditions. Environment and Ecology. 28: (3) 1498-1501.

Singh, M. K. S. Kumar and R. Ram. (2008). Effect of nitrogen and potassium on growth, flowering and bulb production in Asiatic hybrid lily cv. Novecento. $J$. Orna. Hort 11: (I) 45-48.

Sindhu,L.M., and Pathania ,S.(2003). Integrated nutrient management for growth and yield in Glory Lily (Gloriosa superba L.). Journal of Meicinal plant Research. Vol.7(43),pp.3197-3201

Memon, S. M. and Z. Oraghi Ardebili. (2013). Effect of biofertilizers on growth, flowering and yield of gladiolus. The Asian J. Horticulture, 4(1): $285-287$

Nair, S. A. Attri, and B. L. Sharma. (2000). Effect of $\mathrm{N}$ and $\mathrm{P}$ on growth and flowering of tuberose (Poliamhes tuberosa L.). J. Trop. Agri. 38: (1/2) 6668.

Nayak, D. Mandal, T. and Roychowdhury, N. (2005). Effect of NPK nutrition on growth and flowering of (Gerbera 
jamesonii L.) cv. Constance. Orissa J. Hart. 33: (2) 11-15.

Niedziela, C. E., Jr. Kim, S. H. Nelson, P. V. Hertogh, and A. A de. (2008). Effects of N-P-K deficiency and temperature regime on the growth and development of Lilium longiflor "Nellie White' during bulb production under phylotron conditions. Scientia Hort, 116: (4) 430436.

Panday, R.K; Rathore, P, and Singh, M.K. (2001). Effect of different levels of nitrogen and phosphorus an gladiolus under Agra condition. J. Orna. Hort. (New Series), 3(1): 60-61.

Parekh, N. S. Patel, H. C. Sitapara, H. H. Parmar, A. B. and Nayee, D. D. (2010 Response of nitrogen, phosphorus and potash on growth and flower production of chrysanthemum (Chrysanthemum morifolium Ramat)cv. UHR-b. Asian J Hon 5: (1)139-147.

Sehrawat, S. K. Dahiya, D. S. S. Singh and Rana, G. S. (2003) Growth, flowering and corm production in gladiolus as influenced by NPK. Application. Haryana J. Hort. Sci. 32:(3/4) 222-224.

Sankat C.K. and Mujaffar S. (1994). Water Balance In Cut Anthurium Flowers In Storage And Its Effect On Quality. Acta Hort, 368: 723 -732.

Yadav, R.K. and Roy, A. R. (2006). Effect of nitrogen, phosphorus and potassium on growth, flowering ad corm production of gladiolus cv. Pusa Shabnum under Meghalaya conditions. Environment and Ecology 24: (3) 939-94.

\section{How to cite this article:}

Pooja Pahare and Suchismita Mishra. 2020. Effect of NPK on Plant Growth and Quality of Lilium Hybrid (Asiatic Lily) Tresor under Polyhouse and Open Condition. Int.J.Curr.Microbiol.App.Sci. 9(06): 1968-1980. doi: https://doi.org/10.20546/ijcmas.2020.906.243 